\title{
Tarefas pendentes em matéria de inclusão educa- tiva: percepção dos acadêmicos da Universidade Católica do Maule - Chile
}

\author{
Juan Cornejo Espejo \\ Universidad Católica del Maule (Chile)
}

\section{Resumo}

Oartigo é resultado de uma investigação qualitativa que tenta captar as percepções que têm os acadêmicos responsáveis pela formação de professores da Universidade Católica do Maule UCM) em relação à inclusão educativa. A coleta da informação fez-se através de entrevistas semiestructuradas em profundidade e a análise das mesmas seguindo os supostos da teoria fundamentada. A partir da codificação aberta agruparam-se os temas mais recorrentes estabelecendo-se as respectivas correlações na análise relacional referido às percepções dos acadêmicos da UCM. A sua vez na análise seletiva esboçaram-se os principais desafios que esses mesmos acadêmicos visualizam em matéria de inclusão educativa, especialmente aqueles aspectos que podem contribuir a mudar a cultura escolar. As principais conclusões do estudo mostram que embora os académicos da UCM têm ampliado ampliado sua noção de inclusão, sublinhando o caráter emergente do tema, não limitado às necessidades educativas especiais (NEE), identificam importantes obstáculos e nodos críticos tanto no sistema escolar como na própria Universidade.

Palavras-chave: Inclusão Educativa. Problemas. Desafíos. Universidade Católica do Maule.

\section{Pending tasks in the field of inclusive education: perception of acade- mics of the Catholic University of Maule - Chile}

\begin{abstract}
The article is the result of a qualitative research that tries to capture the perceptions of academics responsible for training teachers of Catholic University of Maule (UCM) in relation to educational inclusion. The data collection was done through semi-structured interviews and the analysis thereof on the assumptions of Grounded Theory. From open coding the most recurrent themes were grouped establishing the respective correlations in the relational analysis based on the perceptions of academics from the UCM. In turn, in the selective analysis they outlined the main challenges those same academics from the UCM displayed in educational inclusion, especially those aspects that can contribute to changing the school culture. The main findings show that although they has expanded their notion of inclusion, highlighting the emerging nature of the topic, not limited to special educational needs, identify important obstacles and critical nodes in both the school system and in the University.

Keywords: Inclusive Education. Problems. Challenges. Catholic University of Maule.
\end{abstract}


Tarefas pendentes em matéria de inclusão educativa: percepção dos acadêmicos da Universidade Católica do Maule - Chile

\section{Tareas pendientes en materia de inclusión educativa: percepción de los académicos de la Universidad Católica del Maule - Chile}

\section{Resumen}

El artículo es resultado de una investigación cualitativa que intenta captar las percepciones que tienen los académicos responsables de la formación de profesores de la Universidad Católica del Maule (UCM) en relación a la inclusión educativa. La recolección de la información se hizo a través de entrevistas semiestructuradas en profundidad y el análisis de las mismas siguiendo los supuestos de la teoría fundamentada. A partir de la codificación abierta se agruparon los temas más recurrentes estableciéndose las respectivas correlaciones en el análisis relacional referido a las percepciones de los académicos de la UCM. A su vez en el análisis selectivo se esbozaron los principales desafíos que esos mismos académicos visualizan en materia de inclusión educativa, especialmente aquellos aspectos que pueden contribuir a cambiar la cultura escolar. Las principales conclusiones del estudio muestran que los académicos de la UCM si bien han ampliado su noción de inclusión, subrayando el carácter emergente del tema, no limitado a las necesidades educativas especiales (NEE) identifican importantes obstáculos y nudos

34 críticos tanto en el sistema escolar como en la propia Universidad.

Palabras clave: Inclusión Educativa. Problemas. Desafíos. Universidad Católica del Maule.

\section{Introducción}

La inclusión educativa se ha transformado desde hace ya algún tiempo en un tema de discusión en el contexto de los currículos de formación de profesores. Ello motivado por el reconocimiento de que el sistema escolar es mucho más diverso y al mismo tiempo complejo de lo que se pensaba hasta no hace mucho tiempo. En la escuela es posible identificar estudiantes no sólo con distintos ritmos de aprendizaje o niveles cognitivos, sino también con distintas capacidades físicas, provenientes distintas culturas, con distintos credos religiosos y sistemas valóricos, pertenecientes a minorías étnicas o pueblos originarios de América, con distintas orientaciones sexuales o identidades genéricas entre otras muchas peculiaridades. 
Esta riqueza, tradicionalmente invisibilizada por la escuela en razón de los procesos asimilizadores y homogeizantes que estaban en la base de los sistemas educativos desconocían que la escuela es básicamente una "comunidad de diferencias" (RHOADS, 1994; ARANZAZÚ; AGUADO, 2007). No obstante, esas diferencias han aflorado con más fuerza en el último tiempo, situación que se ha visto reforzada por los flujos migratorios que se han intensificado desde el último cuarto del siglo XX, impulsados por la globalización de la economía y la masificación de los medios de comunicación social.

El fenómeno migratorio presente en los países del primer mundo ya desde la época de post - guerra, se ha tornado más visible en América Latina con el término de las dictaduras militares. Los flujos migratorios que durante décadas se dirigieron, especialmente, hacia los países del primer mundo, dadas las mayores restricciones migratorias impuestas por esos países, la desaceleración de la economía internacional, el mejoramiento de los medios de transporte pero, sobre todo, por la permeabilidad de las fronteras nacionales, las facilidades migratorias y los requerimientos de mano de obra de algunos países latinoamericanos han hecho que muchos de esos flujos se reorienten hacia la misma región.

En ese contexto son numerosos los(as) niños(as) y adolescentes, hijos de migrantes, que deben reinsertarse en los sistemas educativos de los países receptores con la consecuente tensión de conciliación de sus culturas de origen y la cultura nacional que es transmitida por los sistemas escolares que los acogen.

Lo anterior sumado a la expansión de los sistemas educativos en el continente en las últimas décadas ha incidido a que confluyan en la escuela una multiplicidad de requerimientos y demandas de inclusión y reconocimiento de su diversidad.

En este sentido no se puede obviar que pese a los esfuerzos la escuela no sólo tenía un acceso limitado, sino que una de sus funciones prioritarias era la socialización y escolarización basada en la asimilación y homogeneización de todos sus miembros. Ese paradigma pareciera haber entrado en crisis por las nuevas corrientes educativas y el fuerte impulso dado por Unesco en la Conferencia Mundial sobre Educación para Todos, para la satisfacción de las necesidades básicas de enseñanza, llevada a cabo en Jontiem - Tailandia, el año 1990, y el impulso dado a la educación especial a través de la Declaración de Salamanca (1994). Es decir, los nuevos escenarios han terminado imponiendo desafíos y 
tareas a los sistemas educativos regionales, tanto en lo que respecta al acceso y calidad de la educación impartida, como a la inclusión de nuevos grupos, históricamente excluidos e invisibilizados de los sistemas educativos formales.

Papel clave en este proceso de transformación le cupo a la educación especial al colocar en la agenda de discusiones la necesidad de avanzar de la integración escolar a una auténtica inclusión de todos los(as) niños(as) con necesidades educativas especiales (NEE). No obstante, a través de ellos(as) se hicieron visibles otros grupos objetos de exclusión de los sistemas escolares formales. Por lo pronto, entre los beneficiarios están los niños(as) y adolescentes con capacidades físicas distintas, pertenecientes a minorías étnicas o a pueblos originarios.

Y si bien se reconoce que aún queda mucho camino por recorrer en el proceso de inclusión escolar de todos(as) aquellos(as) que por alguna razón han sido marginados, es evidente que ese proceso además de necesario es irreversible. Probablemente, uno de los últimos grupos sobre los cuales hay menos conciencia acerca de la urgencia de ser incluidos desde su peculiaridad, y que se han constituido en un dolor de cabeza para muchos(as) educadores(as) que no saber como lidiar con ellos(as), son los(as) niños y adolescentes disidentes 36 sexuales del ideario heterosexista o cuyas identidades genéricas no son coincidente con los roles de género tradicionalmente asignados.

No sin razón se ha señalado a este grupo como el de más alto riesgo de ser objeto de violencia escolar y bullying homofóbico en la escuela (UNESCO, 2012 l, con el consecuente problema de salud pública por los eventuales daños posológico que el acoso y hostigamiento supone en la autoestima y desarrollo psico - emocional de las víctimas (AMERICAN EDUCATION RESEARCH ASSOCIATION, 2013).

Siendo ese el contexto, la investigación que a continuación se presenta indaga en torno a las percepciones y representaciones que tienen los(as) académicos(as) responsables de la formación de profesores de la Universidad Católica del Maule (UCM) respecto de la inclusión educativa. Ello como un paso previo al análisis de la incorporación efectiva de las problemáticas y cuestionamientos curriculares que supone para la formación de profesores del siglo XXI la inclusión educativa en un sentido más amplio y no limitado a las NEE.

Es importante señalar que los resultados aquí expuestos hacen parte de una investigación mayor acerca del tema. Básicamente la investigación 
constaba de una parte cuantitativa a través de la aplicación de un cuestionario, el cual fue aplicado al $95 \%$ de los académicos(as) responsables directos de la formación de profesores de las facultades de ciencias de la educación, ciencias y ciencias religiosas y filosóficas de la UCM.

Se excluyeron de esa muestra a los(as) académicos(as) que dictan docencia en el Departamento de Educación Especial, dado el mayor grado de envolvimiento y conocimiento acerca del tema. Se excluyeron, asimismo, a los(as) académicos(as) del Instituto de Estudios Generales, pues, si bien imparten docencia a estudiantes de pedagogías, sus cursos son electivos o de formación religiosa; es decir, no guardan relación directa con la formación pedagógica profesional y/o disciplinar.

Luego de aplicado el cuestionario, se optó por profundizar algunos aspectos de los ítemes allí contenidos a través de la aplicación de una entrevista semi - estructurada en profundidad a 12 académicos(as) bajo los mismos criterios de inclusión y exclusión antes señalados. Los resultados de esas entrevistas son las que a continuación se presentan, las cuales fueron analizadas siguiendo los supuestos de la teoría fundamentada.

\section{Consideraciones preliminares}

No se puede obviar el carácter polisémico con que se ha revestido la noción inclusión educativa. Pese a sus múltiples significados la mayor parte de los(as) estudiosos(as) del tema coinciden en que ella se refiere a la:

[...] transformación de las culturas de las escuelas para aumentar el acceso o presencia de todos los estudiantes (no sólo de los grupos marginados o vulnerables), incrementar la aceptación de todo el alumnado, maximizar su participación en múltiples ámbitos de actividad, y aumentar su rendimiento (ARTILES; KOZLESKI; DORN; CHRISTENSEN, 2006, p. 67).

Sin embargo, esa conceptualización que amplía a los posibles beneficiarios de las políticas o acciones inclusivas, no restringiéndolo a los estudiantes vulnerables o pertenecientes a grupos minoritarios, no ha resultado una discusión fácil o libre de tensiones. Por lo pronto, es posible identificar cuatro tendencias que de alguna forma distorsionan el intento de ampliar su compresión: 
1. Históricamente las investigaciones alusivas al tema se han tendido a concentrar en estudiantes con necesidades educativas especiales.

2. Los estudios se han tendido a concentrar en el aula o establecimiento educacional, descuidando las intersecciones de las identidades de los(as) estudiantes (clase social, lengua, género, pertenencia étnica etc.).

3. Ha sido entendida como el lugar donde los(as) estudiantes con necesidades educativas especiales reciben una educación particular en el mismo entorno del aula ordinaria.

4. La mayor parte de las investigaciones, sobre todo las focalizadas en el establecimiento escolar, han tendido a no documentar sus resultados o los alcances de las medidas propuestas (ARTILES; KOZLESKI, 2014).

Otro elemento que explica algunas de las dificultades de arraigo de la inclusión educativa en el ámbito escolar de los países del tercer mundo es que el modelo se originó en los países del primer mundo, esparciéndose luego al resto a través de lo que se denominó una "agenda global". Situación que de alguna forma ha dificultado tanto su compresión como la necesidad de su implementación, en tanto exigencia de una escuela que busca atender sin restricciones a todos (as) los (as) que acceden a ella.

Un hito importante en el fortalecimiento del modelo, fue la Declaración de Salamanca (1994), la cual se basó en el principio de que todos(as) los(as) estudiantes que tuviesen algún requerimiento educativo especial debían ser asistidos(as) y atendidos(as) por los respectivos sistemas educacionales nacionales.

Actualmente existe consenso de que la inclusión educativa está comprometida con una agenda de justicia y equidad, enmarcada en el contexto de los derechos individuales. No obstante, en la práctica se verifica que tanto la producción intelectual en torno al tema, como los(as) responsables de la formación de profesores(as) se distancian de los objetivos originales (ARTILES; KOZLESKI, 2014).

Esto es, los(as) profesores(as) en ejercicio como los que se están formando no cuentan con las competencias o habilidades necesarias para trasmitir a sus estudiantes esas nociones o la capacidad para desarrollar proyectos de intervención que efectivamente pongan en marcha una educación auténticamente inclusiva. 


\section{Método}

El estudio privilegia una metodología de investigación cualitativa, recurriendo a un enfoque inspirado en la teoría fundamentada. En el caso en estudio, el análisis de las percepciones que tienen los académicos(as) de la UCM en relación a la inclusión educativa y los desafíos que esta impone a los sistemas educativos contemporáneos. A partir de las percepciones de los académicos(as) se accede a los significados que esos(as) profesionales le atribuyen al fenómeno en estudio, particularmente a la factibilidad de incluir esta perspectiva teórico pedagógica al currículo de la formación de profesores(as).

\section{Participantes}

Los(as) participantes del estudio son 12 académicos(as) de la UCM, pertenecientes a las facultades de ciencias de la educación, ciencias y ciencias filosóficas y religiosas. Entre los criterios de inclusión se privilegio la paridad de género, profesores(as) directamente relacionados con la formación de profesores, de distintas asignaturas, con más de 5 años de experiencia profesional pertenecientes a 9 de las 10 pedagogías que imparte la UCM. Los criterios de exclusión se focalizaron básicamente en tres aspectos: profesores(as) de la carrera de Educación Diferencial, en razón de que se suponen tienen un conocimiento más acabado del tema en estudio; profesores(as) part - time; y profesores que si bien imparten cursos electivos para estudiantes de pedagogías no están directamente implicados en la formación de profesores(as) (Instituto de Estudios Generales).

\section{Instrumento de recolección de la información}

La recolección de la información se realizó a través de entrevistas semi - estructuradas en profundidad, para lo cual se diseñó un guión preestablecido de preguntas. 


\section{Procedimiento}

Se optó por una estrategia individual de producción de datos, ya que permite indagar respecto de las percepciones y opiniones de los entrevistados(as), sin que ello suponga algún grado de intimidación, favoreciendo la profundidad y riqueza de su relato. Toda la información proveniente de la entrevista fue analizada a través de la Teoría Fundamentada.

\section{Análisis de la información}

La codificación y análisis de la información siguió las tres etapas propuestas por la teoría fundamentada: una codificación abierta, que consiste en un análisis descriptivo de la información; una codificación axial que privilegia un análisis relacional de la información; y una codificación selectiva centrada en la construcción de un modelo o teoría explicativa. La codificación de la información en sus distintas etapas fue triangulada con el propósito de evitar sesgos.

Las opiniones fueron transcritas tal cual fueron emitidas por los(as) entre40 vistados. Los códigos utilizados corresponden al número de la entrevista seguido del párrafo correspondiente.

\section{Consideraciones éticas}

Entre las consideraciones éticas del estudio destaca el carácter "voluntario" de la participación de los entrevistados, no mediando ninguna retribución económica, habiéndoseles garantizando además, no solo el anonimato sino la posibilidad de suspender la entrevista cuando lo desearan, no acarreándole ello ninguna responsabilidad o consecuencia negativa. El material obtenido de las entrevistas fue utilizado única y exclusivamente para los fines declarados en esta investigación. 


\section{Análisis Descriptivo}

\section{Nociones de inclusión educativa}

Todos(as) los(as) académicos(as) entrevistados(as) esbozan algunas ideas de lo que ellos(as) creen es la inclusión educativa. Algunas de esas nociones la relacionan con tolerancia, aceptación, respeto a la diversidad (El, p. 1). Para otros, ella tiene que ver "[...] con acoger a los estudiantes con sus características reales, eh... entendiendo no solamente los alumnos con capacidades educativas especiales sino también a aquellos que tengan opciones distintas o que vengan de culturas diversas, aquellos que tengan como se habla hoy en día, los más talentosos" (EII, p. 1).

Otro(as) de los(as) entrevistados(as) dice que la inclusión "[es mucho más amplio, no se refiere solamente a los alumnos con NEE, también está orientado al resto de la comunidad que forma el grupo curso y está orientado a los adultos que participan en el proceso de inclusión[...]" (Elll, p. 1). Otro(a) entrevistado(a) pone el acento en la inclusión de "[...] los alumnos con capacidades físicas disminuidas y en aulas de clases comunes, como por ejemplo, estos niños con problemas auditivos, que antes tenían sus propios espacios, sus propias escuelas, de cierto momento comienzan a ingresar a establecimientos educacionales normales" (EIV, p. 1).

Prácticamente todos(as) entrevistados subrayan que la inclusión educativa no está limitada a las NEE (la noción clásica aludía únicamente a los(as) estudiantes más descendidos), sino que la misma noción de NEE, a juicio de varios de ellos(as) debería considerar a los(as) estudiantes talentosos(as). "La inclusión no está supeditada, obligada y que no tiene que ser solamente para los niños con NEE" (EIII, p 3). Asimismo, incluyen dentro de esa noción a los(as) estudiantes con capacidades físicas distintas.

La inclusión se asocia también con el aprender a convivir juntos: "Es toda una acomodación una readecuación, es una forma de aprender a convivir juntos" (EVII, p. 8). 


\section{Inclusión educativa no limitada a las NEE}

La inclusión educativa para todos los entrevistados no se limita a los(as) estudiantes con NEE (descendidos). "La inclusión no está supeditada, obligada y que no tiene que ser solamente para los niños con necesidades educativas especiales [... no podemos nivelar hacia arriba al al grupo que estaba descendido y olvidarnos de aquel grupo que destaca, sin brindarles la ayuda que necesita, es necesario atender a ambos" (EVII, p. 25-26).

\section{Inclusión educativa opuesta a la homogeneización}

Para algunos de los(as) entrevistados la inclusión propiamente educativa deberían ser "[...] acciones concretas para integrar la diferencia y tratar de no homogeneizar, sino hacer más bien que todos los alumnos, en este caso la comunidad escolar en general crezca junto, pero respetando la diferencia y que esa diferencia aporta a una comunidad escolar" (EI, p. 2). "En el colegio tu tiendes a la homogeneización tu quieres ojalá que todos sean iguales, que

42 todos se porten igual [...] que todos vayan igualmente vestidos" (EI, p. 27). "Es toda una acomodación una readecuación, es una forma de aprender a convivir juntos" (EIII, p . 2).

\section{Inclusión educativa y normativa vigente}

Algunos(as) de los(as) entrevistados(as) destacan las exigencias legales que existen en materia de inclusión educativa. "Hoy en día a nivel escolar solicitan que exista como dentro del contexto más específico tenemos hasta cuatro estudiantes por aula que son de algunas discapacidades transitorias y dos permanentes eso es en términos de las necesidades educativas especiales hasta ahí conocemos, pero respecto de las otras situaciones como etnia, por ejemplo, no está como definido" (EV, p. 7). 


\section{Confusiones que genera la inclusión}

Para algunos(as) de los entrevistados la inclusión no sólo se ha prestado para confusiones sino que gran parte de ella queda sólo en el discurso. "Yo siento que hoy en día hay mucho de discurso, siento que se puso de moda y en ese contexto hay muchas personas que todavía no consideran, incluso cuesta hacer la diferenciación entre los conceptos de inclusión e integración, inclusión efectiva, inclusión educativa, y no tiene que ver sólo con los niños con necesidades especiales sino que tiene que ver con todos nosotros, tiene que ver con los ritmos de aprendizaje [...]" (EV. p. 12).

\section{Valoración de la diferencia}

Este aparece como uno de los aspectos claves y distintivos de la inclusión educativa. "Claro, porque tú tienes que aprender a valorar lo diferente y a descubrir la riqueza que tienen las individualidades y eso es muy demandante y requiere que tú entiendas los resultados de otra manera, los logros, los objetivos no están solamente basados en números, sino que en otro tipo de valoración". Esa valoración exige además, el tratar de entender al diferente (El, p. 62-63).

\section{Herramientas insuficientes}

Uno de los temas recurrentes en todas las entrevistas es el reconocimiento de que los profesores en ejercicio, y los estudiantes en formación no sólo no fueron preparados para lidiar con la diferencia, sino que tampoco tienen los subsidios suficientes para entenderla. "Creo que a los profesores les falta una herramienta para enfrentar este tipo de alumno en el aula, una que por el tipo de formación que se recibe [...]" (El, p. 6). Esa falta de preparación se expresa en la incapacidad de los(as) profesores(as) de detectar a los estudiantes con NEE. "[...] pero nadie te hablaba a ti, ya tiene déficit atencional, síndrome no sé de qué, no había esa capacidad [...] y siento que ahora aunque hay mayor conocimiento, los profesores no todos contamos con la información como para detectarlo [...]" (El, p. 16). "[...] en la escuela generamos discriminación, al que se porta mal lo mandamos por allá, al que sobresale tampoco estamos preparados, estamos preparados para la normalidad y no estamos preparados para 
los extremos aquel que le falta, aquel que le sobra [...]" (EV, p. 19). Esa falta de preparación tendría que ver, a juicio de uno(a) de los(as) entrevistados(as), con una formación docente no actualizada (EIII, p. 5).

\section{Inclusión educativa tema emergente}

Uno de los consensos en el que parecen converger algunos de los(as) entrevistados(as) es en el reconocimiento de la novedad del tema. "Ahora recién está apareciendo ese tema de la inclusión, es un tema nuevo, no es un tema que se haya tratado como tal antes, yo siento que es un tema relativamente nuevo" (El, p. 26 e p. 28).

\section{Formación docente insuficiente}

Uno de los temas que preocupa a la mayor parte de los(as) entrevistados(as) es la falta de formación académica que evidencian los(as) profesores(as) para atender a la diversidad en el aula y consecuentemente pro-

44 mover la inclusión educativa. "[... ] yo creo que en la formación que estamos brindando todavía está pegada en un currículo profesional, que no considera el decreto 170 y recién está mirando la aplicación de la Ley SEP y los docentes que tienen que atender a los nuevos profesionales no están preparados" (EVII, p. 31 1). "No estamos preparados técnicamente todavía en el nivel que necesitamos para transmitir a nuestros estudiantes, para que ellos lo apliquen en el aula concreta" (EV, p. 4). "Porque los profesores no se encuentran preparados para acoger y en el fondo brindar una buena educación a los estudiantes, porque no están preparados, porque además el colegio tampoco se preocupa y se interesa en prepararse" (EXII, p. 72).

\section{Valores asociados a la inclusión}

Los valores que los(as) entrevistados(as) asocian con la inclusión son el respeto a la diversidad, la aceptación y la tolerancia. "[... ] la inclusión tiene que ver con el tema de la diversidad y no restringirlos a uno que tiene una necesidad 
educativa especial de aprendizaje, también tiene que ver con un tema de cultura diferente, un idioma distinto" (EXI, p. 21 ).

Para una de las entrevistadas la inclusión tiene que ver con el diálogo interreligioso. "[...] mi alumno tiene que aprender el tema del diálogo interreligioso [...] el respeto cultural por toda la riqueza cultural que trae ese alumno a través de su manifestación religiosa, y claro en este sentido tú tienes que considerarlo que también es parte de la inclusión [...]" (El, p. 22). "Creo que interactuar con personas distintas [...] de distintas culturas, con opciones distintas, con necesidades particulares" (Ell, p. 69).

\section{Homosexualidad: tema que complica}

Uno de los temas que aún no han sido bien asimilados en los contextos educativos, a juicio de algunos(as) de los(as) entrevistados, con la inclusión educativa es el de la homosexualidad. Es más, se reconoce que en oportunidades deriva en bullying homofóbico. "[...] en al menos dos o más grupos de los cinco que observe salía el tema de la homosexualidad en broma, se molestan entre ellos y bromean, supuestamente era un futbolista y decían te gusta el arquero y decía sí y todos se reían y como son jóvenes le dijeron lindo, hay perdón linda y todos se reían, me llamó mucho la atención, y aquí hay un tema con eso fuertísimo [...] muy homofóbicos [...] no sé si el rendimiento académico, pero de la persona que en el fondo podía ser discriminada sí por supuesto, tenemos caso de que el bullying lleva a extremos como el suicidio y no es poco frecuente" (EXI, p. $131-1321$. 
Figura 1

Percepción que tienen los(as) académicos(as) de la UCM en relación a la inclusión educativa

\section{Inclusión educativa}
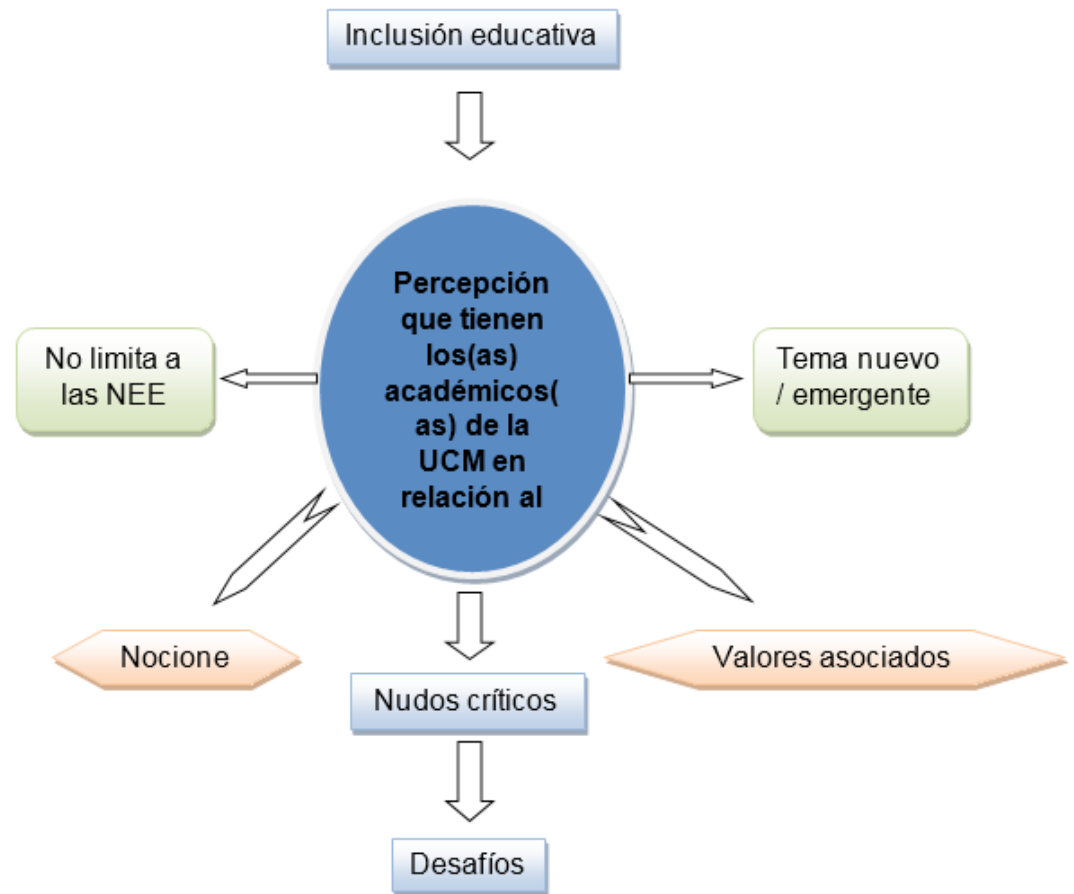

Fuente | creación propia

\section{Análisis relacional: percepción que tienen los(as) académicos(as) de la UCM en relación a la inclusión educativa}

Una primera relación que se puede establecer a partir de las ideas que esbozan los entrevistados en relación a la inclusión educativa es que ella no está limitada a las NEE, entendiendo por tal la atención preferente a los(as) estudiantes descendidos o con problemas de aprendizaje. "No lo restrinjo sólo a las necesidades especiales, sino que lo veo más amplio que tiene que ver con respetar la diferencia de cada persona por su naturaleza única propia de humano" (El, p. 3). 
Esta noción claramente amplía no sólo las nociones subyacentes al tema en estudio sino el espectro de posibilidades de incorporación de nuevos grupos o personas que son objeto de exclusión y/o discriminación de los sistemas escolares formales. Es más, en algunas de las entrevistas la propia noción de NEE incluye expresamente tanto a los(as) estudiantes descendidos como a los(as) talentosos(as); es decir, ambos grupos de estudiantes constituyen una NEE que, a su juicio, debería ser atendida por el sistema escolar.

Esta idea es coincidente con las nuevas corrientes educativas que apuestan por una comprensión ampliada de la inclusión educativa, que inclusive no está restringida a un grupo específico objeto de exclusión, sino a maximizar la participación y rendimiento de todo el alumnado (ARTILES \& KOZLESKI; DORN \& CHRISTENSEN, 2006).

No obstante, lo anterior hay un reconocimiento de que el tema es poco abordado, ya sea por desinterés, ya sea por la falta de preparación de los(as) profesores(as). "Considero que debiera ahondarse bastante en el tema de la inclusión desde distintos puntos de vista no solamente la de tipo de capacidad cognitiva. Va más allá y no está tratada con seriedad yo profundidad que debiera para sembrar integración a nivel del aula y para la vida" (EVI, p. 1).

Otro de las relaciones que atraviesan los relatos de todos(as) los(as) entrevistados(as) es la emergencia y novedad del tema. Prácticamente todos(as) coinciden que es un tema importante, necesario y sobre el cual hay muchas expectativas en razón del poder transformador que tiene la educación en el sentido de tornar las relaciones entre los sujetos en relaciones efectivamente humanas, que asuman la diversidad no como una limitación o un problema sino una riqueza y oportunidad de aprender del otro. "Creo que interactuar con personas distintas, de distintas culturas, con opciones distintas, con necesidades particulares [... ] nos enriquece, no nos retrasa" (EXII, p. 69).

Claramente esta idea es coincidente con las aspiraciones tanto de la literatura especializada como de las exhortaciones que emanan de los documentos de los organismos internacionales, que desde hace algún tiempo vienen promoviendo intensivamente una educación para todos y todas desde una óptica más humanista e inclusiva (UNESCO, 1990; PEREIRA, 2003; ARÁNZAZU \& AGUADO, 2007; BHABHA, 2013; PEREIRA \& MOREIRA, 2014; ARTILES \& KOZLESKI, 2014). 
En esta misma línea cabe consignar que los(as) entrevistados(as) hacen suyos los valores asociados a la inclusión educativa que se desprenden de esas exhortaciones, los cuales apuestan por el respeto a la diversidad, la tolerancia y la aceptación. "[...] porque creo que el aprendizaje es mucho más allá que aprender a sumar, a leer, aprender a restar, multiplicar, sino que tu tienes que aprender la aceptación, aprender el diálogo, la tolerancia, el respeto [...]" (El, p. 5).

Asimismo, las nociones de inclusión educativa que destacan de los dichos de los(as) entrevistados(as) aluden, en primer término como ya se mencionó, a la atención de las NEE tanto de los(as) estudiantes descendidos como de los(as) talentosos(as), pero también se hace referencia explícita a los(as) estudiantes con distintas capacidades físicas, sordos, no videntes. "[...] incluir los alumnos con capacidades físicas disminuidas y en aulas de clases comunes, como por ejemplo estos niños con problemas auditivos, con ceguera, minusválidos, que antes tenían sus propios espacios, sus propias escuelas [...]" (EIV, p. 1). Además, del reconocimiento de la diversidad cultural y lingüística. "[...] también tiene que ver con un tema de cultura diferente, un idioma distinto" (EXI, p. 21). Y aún se reconoce que dentro de esa conceptualización cabe el diálogo 48 interreligioso (El, p. 22).

Para algunos(as) de los entrevistados(as) su apuesta es por colegio donde todos(as) puedan convivir en su diversidad y no en colegios diferenciados o especiales. "Si uno piensa el concepto de educación desde una concepción integral, sin duda, esos niños tienen que estar en un colegio común y corriente donde están todos porque así tu también aprendes a valorar el respeto" (El, p. 20).

Con todo, esa ampliación de la idea de inclusión educativa no desconoce los trabas que obstaculizan la inclusión tanto en la escuela como en la propia Universidad. Por lo pronto, se reconoce que uno de los principales obstáculos es la falta de preparación de los(as) futuros(as) profesores(as) en el tema. "[...] los profesores no siempre están capacitados para enfrentar este tipo de alumnos, entonces como no sabe como trabajar con ellos pueden descuidar todo el curso para tratar de atender esta necesidad y como no tienen esta herramienta es un poco difícil [...]" (EI, p. 4).

Es de decir, los(as) estudiantes de pedagogías de la UCM no sólo no están sensibilizados acerca de los alcances de la inclusión y consecuentemente 
desconocen el tema, sino que en sus prácticas profesionales no saben lidiar con distintos tipos de estudiantes y las problemáticas asociadas a esa diversidad.

Esa falta de competencias para lidiar con la diversidad guarda directa relación, a juicio de los(as) entrevistados(as), con la formación deficitaria que reciben esos(as) futuros(as) profesores(as) en la Universidad. "Creo que a los profesores les falta una herramienta para enfrentar este tipo de alumnos en el aula, una que por el tipo de formación que se recibe [...]" (EXI, p. 6). "No estamos preparados técnicamente todavía en el nivel que necesitamos para transmitir a nuestros estudiantes, para que ellos lo apliquen en el aula concreta" (EV, p. 3).

No obstante, se reconoce también que de parte de los(as) académicos(as) responsables de la formación de profesores(as) que hay confusión conceptual y falta de competencias para el trabajo en inclusión educativa. "Yo creo que la formación que estamos brindando todavía está pegada a un currículo tradicional, en un currículo que no considera el decreto 170 y recién está mirando la aplicación de la Ley SEP y los docentes que tienen que formar a los nuevos profesionales no están preparados" (EIII, p. 11).

Otro obstáculo denunciado por los(as) entrevistados(as) es que pese a los discursos oficiales de las autoridades de gobierno en el sentido de promover la inclusión en las escuelas, los criterios de evaluación continúan privilegiando resultados cuantificables. "Entonces todo es como para lograr objetivos [...] metas cuantitativas [...]" (El, p. 17). "[...] es difícil cuando hay tanta exigencia de resultado, que genera mucha competencia y que por medio hay recursos, el tema económico, sin duda, eso crea el tipo de estudiante que tu quieres hacer y si tu quieres hacer algún tipo de inclusión no va asociada a cosas muy específicas" (EIX, p. 34).

Sin contar con la homogeneización escolar que desvirtúa cualquier intento inclusivo (El, p. 2; p. 27; Elll, p. 2), y la falta de adecuación de la ley a las distintas realidades escolares. "La normativa vigente no hace mucho no era aplicable [... ]. La verdad es que solamente mirando el tiempo en que la Ley fue promulgada, el tiempo que está asumida por los profesores y los establecimientos educacionales, el tiempo en que se ha demorado en cambiar el currículo a una visión más integradora, podría decir que casi con seguridad, que no estamos preparados" (EIII, p. 8).

Otro de los nudos críticos que visualizan algunos(as) de los(as) entrevistados(as), que además de un obstáculo se constituye en un elemento 
que tensiona la inclusión educativa, especialmente en el ámbito de las prácticas pedagógicas es la homofobia, la cual que no está limitada al espacio escolar sino que está instalada en la propia Universidad. "[...] una vez hablaban en consejo de profesores en párvulos, hablaban por ejemplo, tenemos un problema porque tenemos una chica que es lesbiana, pero el problema no es ese, sino que con su pareja se dan la mano, se hacen cariño [...] y para ese cuerpo docente ese era un problema y una complicación, eso debe ser hace como tres años atrás [...]" (Ell, p. 126-127)". "Somos homofóbicos totales, en nuestros discursos seguimos haciendo chistes homosexuales, nos parece gracioso [...]" (Ell, p. 128).

Pese a toda la información hoy disponible aún en algunos relatos de algunos(as) entrevistados(as) sigue apareciendo ideas asociadas a anormalidad. "Mira antes también el mundo homosexual [...] trataban de esconderse, por lo prohibido, no se decía nada porque estaba oculto, donde lo heterosexual era lo único que se aceptaba. Hoy en día estamos en un mundo donde todas esas anomalías, todo este tipo de opciones son defendidas, incluso ahora ya no da miedo decirlo en algunos contextos [... ]" (El, p. 45).

La explicación a esa homofobia es atribuida al emplazamiento de la 50 Universidad, en pleno Valle Central de Chile. Es decir, el machismo asociado al mundo campesino sería la justificación de esa actitud. "[...] porque es un mundo rural, de campesinos [...] un mundo machista, y es por eso yo creo ha contribuido a que haya menos aceptación de las diferencias, de sexualidades" (El, p. 50).

Probamente, estos discursos evidencian que si bien hay una ampliación de las nociones de inclusión, ellas sólo alcanzan a todos los grupos o personas que son objetos de discriminación o exclusión de los sistemas escolares formales. Situación que además de un desafío se convierte en una necesidad si se quiere formar profesores(as) con un nuevo sentido ético de las relaciones humanas.

\section{Análisis selectivo: tareas pendientes en materia de inclusión educativa en la óptica de los(as) académicos(as) de la UCM}

Cómo contribuir a través de la formación de profesores(as) a cambiar la cultura escolar desde una perspectiva de la inclusión educativa es la gran 
tarea que visualizan los(as) académicos(as) de la UCM en los próximos años. En esa tarea consideran clave el papel que la propia Universidad puede hacer tanto desde el punto de vista curricular y de sus prácticas pedagógicas, como de su proyecto educativo. "[...] si tu ves los proyectos de la Universidad, donde aparece la misión, la visión apostamos por la integración, apostamos también por la formación integral de la persona, y eso significa aceptar a la persona con todo lo que es, con sus valores, con sus opciones, con sus creencias y con su sexualidad [...]" (EXI, p. 51 ).

Es decir, la inclusión educativa para los(as) entrevistados(as) supone el desarrollo de políticas y estrategias específicas por parte de la institución, pero también mucha iniciativa y creatividad de los(as) propios(as) académicos(as). "[...] siempre el académico tiene la posibilidad de instalar ciertas temáticas es parte de su libertad de ir más allá, de lo que el programa te plantea. Y en ese sentido, yo como docente he tratado de incorporar ciertas temáticas como diversidad cultural, étnica, la diversidad de aprendizaje, como la de talentos académicos que no está contemplada en la educación básica [...]. Pero por una iniciativa propia no porque esté normado y reglamentado [...]. Los programas de estudio siempre están sujetos a revisión [...]" (EVII, p. 3).

Sin desconocer los obstáculos que entraban la inclusión educativa, y que apuntan, principalmente, a una formación pedagógica deficitaria para atender la diversidad en el aula, a una legislación aún anclada en la visión clásica de las NEE (decreto 170) y ciertos nudos críticos que guardan relación con la homofobia cultural, que aún prevalece en ciertos sectores del profesorado que les dificulta entender que los estudiantes LGBT (lesbianas, gays, bisexuales, transexuales y transgéneros) son también sujetos de la inclusión educativa, se reconoce que la Universidad es un espacio privilegiado para formar en la aceptación. "[...] yo creo que la escuela, la universidad, el instituto es un espacio donde tu puedes ir formando hacia la aceptación, hacia la inclusión, pues, por ley no existen los cambios, sino que los cambios vienen por una convicción profunda [...]" (EXI, p. 56). Esta idea deviene del reconocimiento de que la propia Universidad es "un mundo donde se da la diversidad [...]" (El, p. 54), y que lentamente se está avanzando hacia la aceptación (EI, p. 55). Además de la necesidad de un cambio de mentalidad de los(as) profesores(as). "Porque tu tienes que aprender a valorar lo diferente y a valorar las riquezas que tienen las individualidades y eso es muy demandante, y requiere que tu entiendas los resultados de otra manera, los logros, los objetivos. No están solamente basados en 
números, sino en otro tipo de valoración" (El, p. 62). Todo esto nace, sin duda, del esfuerzo por entender lo diferente (El, p. 63).

A partir de ese diagnóstico, los(as) entrevistado(as)s señalan que la gran deuda de la UCM es la urgencia de un cambio en el modelo formativo; pues, sin él no habrá un cambio efectivo. "Yo creo que no estamos formando para la inclusión, porque tampoco nosotros fuimos formados para la inclusión; de alli, que sea necesario cambiar el modelo formativo" (EXII, p. 17).

Esta convicción deviene del reconocimiento de que la inclusión educativa para los(as) académicos(as) de la UCM es más bien teórica y no una práctica cotidiana o una exigencia curricular. "Yo creo que la conocen más bien a nivel teórico, porque a mí, por ejemplo, me llamó la atención en un postítulo en que participé uno de los objetivos transversales era que tenía que estar siempre incluido como eje el tema de la inclusión [... . La persona que vino a hablar de inclusión se dedicó a mostrarnos definiciones, decretos de lo que era la inclusión y lo que decía el gobierno. Yo dije aquí estamos en el nivel de la teoría, siento que no han bajado a la práctica" (Ell, p. 140-141).

Lo anterior explicable, probablemente, por la falta de preparación y

52 de estrategias metodológicas para la puesta en práctica por parte de los(as) propios(as) académicos(as) de la UCM, que si bien se muestran sensibles al tema señalan no tener todas las herramientas necesarias; además de evidenciar confusiones conceptuales. "Aquí en la Universidad hay un sesgo cuando hablamos de integración. Cuando hablamos de integración hablamos de alguna normativa vigente en relación a la integración, siempre miramos para la educación especial [...]" (EVII, p. 32). "[...] porque nunca estuvimos preparados (...), no nos formamos en esta área de desempeño y luego nos vimos obligados a incorporar entonces, yo creo que más que inclusión estamos haciendo integración [...]. No estamos preparados técnicamente todavía en el nivel que necesitamos para transmitir a nuestros estudiantes, para que ellos lo apliquen en el aula concreta" (EV, p. 2; p. 4).

En otras palabras, aún subsisten confusiones y no se reconocen los alcances e implicancias prácticas, entre inclusión e integración, inclusión y asimilación entre otras ideas y conceptos. Sin contar que el compromiso con el tema viene dado por una actitud personal y no una política definida por la institución. "Yo diría que no, porque el desarrollo de las competencias y las habilidades para manejar, tratar de actuar frente al currículo o frente a los alumnos 
que tienen estas necesidades, generalmente, hasta ahora, el perfeccionamiento corre por cuenta propia" (EVII, p. 33).

No obstante, el reconocimiento de las deudas en la formación de profesores, existe la convicción de que la UCM puede ser un espacio privilegiado de acogida y aceptación de la diversidad. "Nosotros tenemos hoy en día la primera generación [...] y no había gente formada. Yo creo que la Universidad es un espacio donde tu puedes ir formando hacia la aceptación, hacia la inclusión [...]" (EXI, p. 56).

Todos estos desafíos son asumidos a la espera de que las nuevas generaciones de profesores(as) no repitan prácticas discriminatorias, pero sobre todo, rompan con aquellos paradigmas que justifican la exclusión o promueven, de acuerdo a la conceptualización de Bader Sawaia (1999), una "inclusión perversa". "Yo confío que nuestros estudiantes no van a repetir ese modelo. Yo creo que si van a hacer algo si ven una situación así" (Ell, p. 103).

Todo ello además, en vista de instalar estos temas en la escuela, tanto a nivel de la discusión como de las prácticas en el aula. "Exacto que estén capacitados para poner en discusión en el colegio. Si el colegio no tiene un programa de integración, al menos, motivarlos a abrirse para tenerlo" (Ell, p. 104).

En suma, se puede decir que para los(as) académicos(as) de la UCM la inclusión educativa representa no sólo la posibilidad de contribuir a cambiar la cultura escolar desde la formación de profesores(as), sino también la posibilidad de un cambio de la propia mentalidad, de modo de tornarla más abierta y receptiva a la diversidad. 
Artigo

Figura 2

Tareas pendientes en materia de inclusión educativa en la óptica de los(as) académicos(as) de la UCM
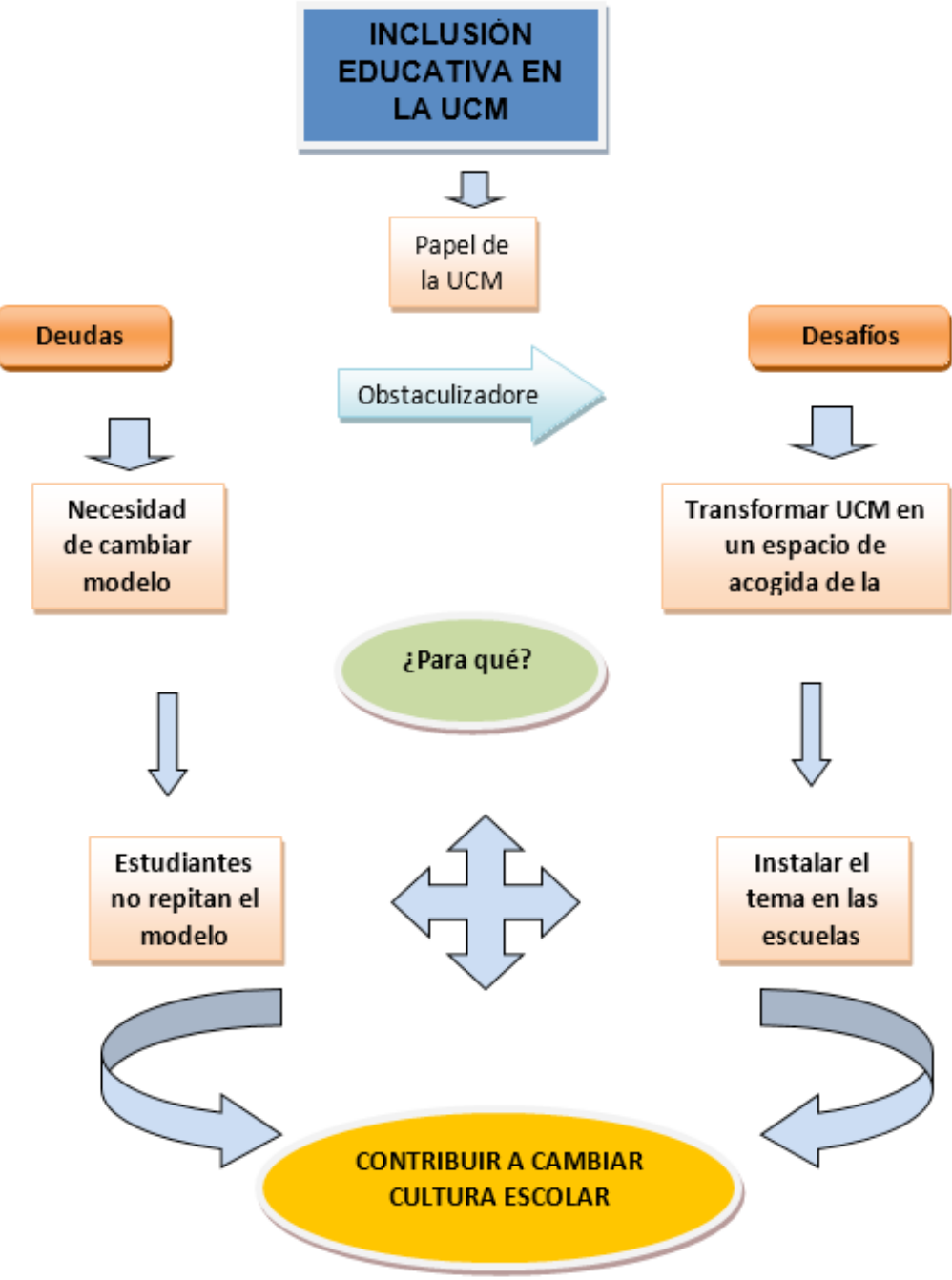

Desafíos

Fuente | creación propia 


\section{Discusión}

En los discursos de los(as) académicos(as) de la UCM aparece reiteradamente la idea de inclusión educativa, ello probablemente motivado por la convicción de que todo sistema escolar abriga una multiplicidad de diferencias. No sin razón, contradiciendo las ideas que prevalecían en el pasado que privilegiaban la homogeneización, la asimilación y la negación de las diferencias (CORNEJO, 201 2), se sostiene en la actualidad que la escuela es "una comunidad de diferencias" (ARÁNZAZU \& AGUADO, 2007).

La sociedad y la escuela, pero sobre todo los profesores, de acuerdo a esa nueva conceptualización, deben estar preparados y capacitados para convivir y lidiar con la diferencia (FELTRIN, 2011 ). Ello supone para las instituciones formadoras de profesores(as) la exigencia de proporcionar los recursos humanos y materiales necesarios para que esa aspiración sea posible. En otras palabras, incluir la diversidad desde una perspectiva amplia como propone Tony Booth \& Mel Ainscow (2002).

Con todo, como se desprende de los testimonios de los(as) entrevistados, si bien existe esa convicción en términos generales, como sostiene Alfredo Artiles \& Elizabeth Kozleski (2014), aún subsisten ideas contradictorias en relación a la inclusión educativa, especialmente en lo que dice relación con la posibilidad de incluir a grupos sobre los cuales no existe plena convicción de que sean objeto de exclusión, como son por ejemplo, los(as) estudiantes LGBT, disidentes sexuales del orden heteronormativo. En este caso aún subsisten inconscientemente ideas asociadas a patología, antinaruralidad o anormalidad, situación que dificulta visualizar a estos jóvenes y adolescentes como grupo susceptible de ser incluido en los sistemas educativos formales sin desconocer, omitir, negar o pretender invisibilizar su particularidad.

En la mayor parte de los entrevistados prevalece la idea de inclusión de los(as) estudiantes con NEE, con capacidades físicas distintas, provenientes de pueblos originarios o migrantes. Incipientemente se asoma el tema de la tolerancia religiosa como elemento que debe ser tenido en consideración a la hora de hablar de inclusión educativa, los(as) estudiantes talentosos o superdotados como una expresión inversa de las NEE y las minorías étnicas.

No se puede obviar tampoco que en esos discursos al hablar de inclusión educativa siempre remiten a grupos cuyos derechos son desatendidos o 
vulnerados. Aún no está afincada la idea de inclusión en el sentido de busca incrementar la aceptación de todos(as) los(as) estudiantes, maximizar su participación en el ámbito escolar y mejorar su rendimiento (ARTILES, KOZLESKI, DORN \& CHRISTENSEN, 2006). Es decir, aún no se entiende la inclusión educativa como una construcción que busca por todos los medios posibles minimizar los procesos de exclusión, maximizando la participación de todos(as) los(as) estudiantes de modo de tornarlos en protagonistas de sus procesos de aprendizaje (PEREIRA \& MOREIRA, 2014).

Otros aspectos que aún permanecen invisibilizados en los(as) académicos(as) de la UCM son las intersecciones de identidades, esto es, un(a) estudiante o un grupo de ellos(as) no sólo es portador de una identidad que motiva la exclusión. Normalmente se superponen otras identidades /clase, lengua, género, etc.) que no pocas veces, refuerzan esa exclusión inicial.

Asimismo, las apreciaciones en relación al tema no están fundadas en estudios o experiencias en aula sino en opiniones, lo que le resta fuerza a sus discursos. Sumado al propio reconocimiento de los(as) académicos(as) de la UCM que señalan que pese a estar sensibilizados no cuentan con la preparación y las herramientas teorico - prácticas para transmitir a los(as) futuros(as)

56 profesores(as) los elementos que les permitan implementar programas inclusivos efectivos en las escuelas.

Probablemente, el avance más relevante sea el abandono de la asociación entre inclusión educativa y NEE. Prácticamente todos(as) los(as) entrevistados(as) insistieron en que la inclusión no puede reducirse a la atención de los(as) estudiantes con problemas de aprendizaje. A su juicio, la inclusión educativa necesariamente debía considerar, además de las nociones convencionales, aspectos culturales, étnicos, lingüísticos, entre otros.

Esta apertura, sin duda, depara a estos(as) académicos(as) con la incerteza y provisionalidad que implica construir el conocimiento en consideración a los distintos contextos y sus significados, situación que presupone asumir el proceso pedagógico con objetivos y estrategias diferenciadas. Desafío derivado a su vez de los procesos políticos, económicos y sociales de la sociedad globalizada y de las exigencias que se derivan de ella.

En ese escenario la búsqueda de nuevos currículos educacionales, las exigencias que impone la formación de profesores(as) y las propuestas de transversalidad de temas controvertidos ponen de manifiesto que los sistemas 
educacionales contemporáneos se encuentran en búsqueda de alternativas formativas tanto para los propios formadores como para los(as) estudiantes (KRONBAUER \& SIMIONATO, 2010).

No se puede obviar tampoco que la inclusión educativa conlleva en esa sociedad globalizada la exigencia ética, como se afirmó en relación a la urgencia en vista de la satisfacción de las necesidades básicas de enseñanza en la Conferencia Mundial sobre Educación para Todos en Jontiem - Tailandia (UNESCO, 1990), que todos son capaces y tienen el derecho de aprender.

De igual modo, que todo proceso educativo que pretende ser realmente inclusivo debe considerar tres dimensiones ineludibles: la construcción de una cultura inclusiva, el desarrollo de políticas específicas y de prácticas efectivamente inclusivas (BOOTH \& AINSCOW, 2002; PEREIRA \& PAULINO, 2014).

A la luz de estos antecedentes y de las propias entrevistas se concluye entonces que las tareas pendientes y desafíos futuros en la UCM, en tanto institución formadora de profesionales, pero de modo especial de profesores(as), en materia de inclusión educativa, sea pasar de la etapa de sensibilización y concientización al desarrollo de una cultura inclusiva al interior de la Universidad que incluya a todos y a todas no importando sus capacidades, orientaciones o peculiaridades, de modo de evitar los discursos desprovistos de contenido o la tentación de una pseudo inclusión o una "inclusión perversa" (SAWAIA, 1999).

El desarrollo de políticas institucionales específicas que hagan posible el florecimiento de esa cultura es otro los aspectos a considerar; pero sobre todo su puesta en práctica, tanto en el currículo como en las prácticas cotidianas de aula. Todo cual contribuirá, sin duda, a cambiar la cultura escolar en un país, y en especial en una región donde se emplaza la UCM, en que aún la inclusión educativa aparece en el horizonte más como una aspiración que una práctica real.

\section{Nota}

$1 \quad$ Fondecyt Regular 1130501. 


\section{Referências}

AMERICAN EDUCATIONAL RESEARCH ASSOCIATION. Prevention of Bullying in Schools, Colleges and Universities. Washington, DC: American Educational Research Association, 2013

ARÁNZAZU, Rosario y AGUADO, María Teresa. Pedagogía de la diversidad. Madrid: UNED, 2007.

ARTILES, Alfredo; KOZLESKI, Elizabeth; DORN, Sherman, \& CHRISTENSEN, Carol Learning in inclusive education research: Re-mediating theory and methods with a transformative agenda. Review of Research in Education, Washington, v. 30, p. 65-108, 2006.

ARTILES, Alfredo y KOZLESKI, Elizabeth. Educación inclusiva en el siglo XXI: notas para un programa de investigación histórico - cultural. In CARDONA, María Cristina y CHINER Esther (Ed.). Investigación educativa en escenarios diversos, plurales y globales. Alicante: AIDIPE - Universidad de Alicante, 2014. p. 51-65.

$\mathrm{BHABHA}$, Homi K. Nuevas minorías, nuevos derechos. Buenos Aires: Siglo XXI Editores, 2013.

58 BOOTH, Tony \& AINSCOW, Mel. Index for Inclusión - developing learning and participation in schools. Bristol: CSIE, 2002.

CORNEJO, Juan. Educación, interculturalidad y ciudadanía. Educar em Revista, Curitiba, n. 43. p. 239-254. jan./mar. 2012.

El. Entrevista. Talca (Región del Maule), 10 jun. 2015.

Ell. Entrevista. Talca (Región del Maule), 12 jun. 2015.

Elll. Entrevista. Talca (Región del Maule), 15 jun. 2015.

EIV. Entrevista. Talca (Región del Maule), 17 jun. 2015.

EV. Entrevista. Talca (Región del Maule), 19 jun. 2015.

EVI. Entrevista. Talca (Región del Maule), 24 jun. 2015.

EVII. Entrevista. Talca (Región del Maule), 29 jun. 2015.

EVIII. Entrevista. Talca (Región del Maule), 6 jul. 2015.

EIX. Entrevista. Talca (Región del Maule), 8 jul. 2015. 
EX. Entrevista. Talca (Región del Maule), 27 jul. 2015.

EXI. Entrevista. Curicó (Región del Maule), 29 jul. 2015.

EXII. Entrevista. Curicó (Región del Maule), 30 jul. 2015.

FELTRIN, Antonio Efro. Inclusão social na escola: quando a pedagogía se encontra com a diferença. 5. ed. São Paulo: Edições Paulinas, 2011.

KRONBAUER, Selenir Corrêa Gonçalves e SIMIONATO, Margareth Fadanelli. Formação de professores: abordagens contemporâneas. 2. ed. São Paulo: Paulinas, 2010.

SANTOS, Mônica Pereira dos. $\bigcirc$ papel do ensino superior na proposta de uma educação inclusiva. Revista Movimento - Revista da Faculdade de Educação da UFF, Niterói, n. 7. p. 78-91, maio, 2003.

SANTOS, Mônica Pereira dos; PAULINO, Marcos Moreira. Inclusão em educação: culturas, políticas e práticas. 2. ed. São Paulo: Cortez, 2014.

RHOADS, Robert. Coming out in college. The struggle for a queer identity. Westport, Connecticut: Bergin \& Garvey, 1994.

SAWAIA, Bader (Org.). As artimanhas da exclusão - analise psicosocial e ética da desigualdade social. Petrópolis: Vozes, 1999.

UNESCO. Conferencia Mundial sobre Educación para Todos: Satisfacción de las necesidades básicas de aprendizaje. New York, UNESCO, 1990. Disponible en: http://www. unesco.org/education/pdf/JOMTIE_S.PDF. Acceso en: 10 feb. 2016.

Declaración de Salamanca y marco de acción para las necesidades educativas especiales. Salamanca: Unesco, 1994. Disponible en: http://www.unesco.org/education/pdf/SALAMA_S.PDF. Acceso en: 10 feb. 2016.

Respuesta del sector educación frente al bullying homofóbico.
Cuadernillo 8. París: Unesco, 2012. Disponible en: http://unesdoc.unesco.org/ images/0022/002229/222918S.pdf. Acceso en: 10 feb. 2016. 
Artigo

Tarefas pendentes em matéria de inclusão educativa: percepção dos acadêmicos da Universidade Católica do Maule - Chile

Prof. Dr. Juan Cornejo Espejo

Universidad Católica del Maule

Facultad de Ciencias de la Educación

Programa de Postgrado en Educación

Grupo de Investigación | Centro de Investigaciones Pedagógicas | Universidad

Católica del Maule | Núcleo Inclusión Educativa

E-mail | jcornejo@ucm.cl

Recebido 9 jun. 2016

Aceito $1^{\circ}$ ago. 2016 